\title{
Comment on the homogeneous nanofluid models applied to convective heat transfer problems
}

Received: 20 June 2011 / Published online: 7 September 2011

(C) Springer-Verlag 2011

\begin{abstract}
In several recent papers, the heat transfer characteristics of nanofluids have been investigated by simply replacing the transport coefficients of the base fluid by the effective transport coefficients of the nanofluids. The present note emphasizes, however, that the governing equations of these homogeneous nanofluid models (in which the velocity-slip effects of the nanoparticles are neglected) can be reduced with the aid of elementary scaling transformations to the respective equations of the regular fluids. Thus, the corresponding nanofluid results can be recovered from the solutions of already solved regular problems by simple arithmetic operations, without any additional research effort. This feature is illustrated here by the specific examples of the classical Blasius and Sakiadis forced convection heat transfer problems.
\end{abstract}

\section{Introduction}

A "nanofluid" consists of a usual liquid, referred to as "base fluid", in which solid particles of submicronic size of 1-100 nm ("nanoparticles") have been dispersed. The nanofluids possess a significantly higher thermal conductivity and single-phase heat transfer coefficients than the respective base fluids. According to the nonhomogeneous equilibrium model proposed by Buongiorno [1], the abnormal increase of the heat transfer characteristics of the nanofluids is due to the velocity-slip of the nanoparticles with respect to the base fluid. The two main effects responsible for the velocity-slip are the Brownian diffusion and the thermophoretic diffusion of the nanoparticles [1]. Without taking into account these two effects, the enhanced heat transfer characteristics of the usual nanofluids cannot be understood. Accordingly, several classical heat transfer problems in clear fluids [2-6] as well as in porous media [7-9] have been reconsidered recently for the case of nanofluids using the nonhomogeneous equilibrium model of Boungiorno [1]. In contrast to the investigations [2-9] based on the nonhomogeneous model [1], in the current year in a number of papers [10-17] homogeneous nanofluid models have been used in which all the velocity-slip effects of the nanoparticles are omitted. The homogeneous nanofluid models adopted in [10-17] are based on the classical balance equations of the regular fluids in which the transport coefficients are simply replaced by the effective transport coefficients of the nanofluids. The aim of the present note is to show that the governing equations of the homogeneous nanofluid models can be reduced with the aid of elementary scaling transformation to the respective equations of the regular fluids. Accordingly, the nanofluid results obtained from the homogeneous models can be recovered from the solutions of already solved regular problems by simple arithmetic operations. This feature is illustrated below by the specific examples of Blasius and Sakiadis nanofluid flows. 


\section{The Blasius and Sakiadis nanofluid problems}

In a recent paper of Ahmad et al. [10], the nanofluid counterparts of the classical Blasius and Sakiadis forced convection heat transfer problems have been investigated using the homogeneous nanofluid model governed by the continuity, momentum and energy equations

$$
\frac{\partial u}{\partial x}+\frac{\partial v}{\partial y}=0, \quad u \frac{\partial u}{\partial x}+v \frac{\partial u}{\partial y}=\frac{\mu_{\mathrm{nf}}}{\rho_{\mathrm{nf}}} \frac{\partial^{2} u}{\partial y^{2}}, \quad u \frac{\partial T}{\partial x}+v \frac{\partial T}{\partial y}=\alpha_{\mathrm{nf}} \frac{\partial^{2} T}{\partial y^{2}} .
$$

Here, all the symbols possess the usual meaning and the subscript nf stands for "nanofluid". Introducing the dichotomic variable $\varepsilon$ with the value $\varepsilon=0$ for the Blasius and $\varepsilon=1$ for the Sakiadis flow, the boundary conditions considered in [10] can be written in the compact form $u=\varepsilon U, v=0$ on $y=0, u \rightarrow(1-\varepsilon) U$ as $y \rightarrow \infty$ where $U$ denotes the uniform free stream velocity in the Blasius case and the uniform surface velocity in the Sakiadis case, respectively. It is assumed that the surface temperature $T_{w}$ is constant in both cases and differs from the ambient temperature $T_{\infty}$ of the nanofluid. Thus, the thermal boundary conditions are the same in both cases and read $T=T_{w}$ on $y=0, T \rightarrow T_{\infty}$ as $y \rightarrow \infty$. Following the work of Tiwari and Das [18], for the thermophysical properties of the base fluid (subscript $\mathrm{f}$ ), of the suspended solid nanoparticles (subscript s) and of the composite nanofluid suspension (subscript nf), in [10] the following relationships have been adopted:

$$
\begin{aligned}
& \mu_{\mathrm{nf}}=\frac{\mu_{\mathrm{f}}}{(1-\varphi)^{2.5}}, \quad \rho_{\mathrm{nf}}=(1-\varphi) \rho_{\mathrm{f}}+\varphi \rho_{\mathrm{s}}, \quad \alpha_{\mathrm{nf}}=\frac{k_{\mathrm{nf}}}{\left(\rho C_{p}\right)_{\mathrm{nf}}}, \\
& \frac{k_{\mathrm{nf}}}{k_{\mathrm{f}}}=\frac{k_{\mathrm{s}}+2 k_{\mathrm{f}}-2 \varphi\left(k_{\mathrm{s}}-k_{\mathrm{f}}\right)}{k_{\mathrm{s}}+2 k_{\mathrm{f}}+\varphi\left(k_{\mathrm{s}}-k_{\mathrm{f}}\right)}, \quad\left(\rho C_{p}\right)_{\mathrm{nf}}=(1-\varphi)\left(\rho C_{p}\right)_{\mathrm{f}}+\varphi\left(\rho C_{p}\right)_{\mathrm{s}} .
\end{aligned}
$$

In the above equations, $\varphi$ denotes the volume fraction of the nanoparticles in the nanofluid. With the usual definition of the stream function, $u=\partial \psi / \partial y, v=-\partial \psi / \partial x$, and with the aid of the similarity transformations specified by their Eq. (10), the authors of [10] reduce the above boundary value problems to the form

$$
\begin{aligned}
& \frac{1}{(1-\varphi)^{2.5}\left(1-\varphi+\varphi \rho_{\mathrm{s}} / \rho_{\mathrm{f}}\right)} f^{\prime \prime \prime}+\frac{1}{2} f f^{\prime \prime}=0, \\
& \frac{1}{\operatorname{Pr}_{\mathrm{f}}} \frac{\left(k_{\mathrm{nf}} / k_{\mathrm{f}}\right)}{\left[1-\varphi+\varphi\left(\rho C_{p}\right)_{\mathrm{s}} /\left(\rho C_{p}\right)_{\mathrm{f}}\right]} \theta^{\prime \prime}+\frac{1}{2} f \theta^{\prime}=0, \\
& f(0)=0, \quad f^{\prime}(0)=\varepsilon, \quad f^{\prime}(\infty)=1-\varepsilon, \\
& \theta(0)=1, \quad \theta(\infty)=0,
\end{aligned}
$$

where $P r_{\mathrm{f}}=v_{\mathrm{f}} / \alpha_{\mathrm{f}}$ is the Prandtl number of the base fluid and the prime denotes differentiations with respect to the similarity independent variable $\eta$. Subsequently, in [10] a comprehensive parameter study of the boundary value problems (3)-(6) for water-based nanofluids containing respectively $\mathrm{Cu}, \mathrm{Al}_{2} \mathrm{O}_{3}$ and $\mathrm{TiO}_{2}$ nanoparticles has been reported. The results for the skin friction coefficient $C_{f}$ and the local Nusselt number $N u$ have been given in [10] in terms of the dimensionless wall shear stress $f^{\prime \prime}(0)$ and the dimensionless wall temperature gradient $\theta^{\prime}(0)$ according to the equations

$$
\operatorname{Re}_{x}^{1 / 2} C_{f}=\frac{f^{\prime \prime}(0)}{(1-\varphi)^{2.5}}, \quad R e_{x}^{-1 / 2} N u=-\frac{k_{\mathrm{nf}}}{k_{\mathrm{f}}} \theta^{\prime}(0)
$$

where $R e_{x}=U x / v_{\mathrm{f}}$ is the local Reynolds number of the base fluid.

\section{The rescaled boundary value problems}

A quick inspection of the governing equations (3) and (5) (which are Eqs. (11) and (12) of [10]) could raise the impression that the nanofluid model applied in [10] would differ essentially from the corresponding equations of the regular fluids, and thus, in spite of the neglected velocity-slip effects in the energy equation, it could possess a new physical content. However, the model based on Eqs. (3) and (5) is fully equivalent to that of the regular viscous fluids. Indeed, with the aid of the scaling transformations of the independent and dependent variables 


$$
\eta=\sqrt{a} \xi, \quad f(\eta)=\sqrt{a} F(\xi)
$$

where

$$
a \equiv \frac{v_{\mathrm{nf}}}{v_{\mathrm{f}}}=\frac{1}{(1-\varphi)^{2.5}\left(1-\varphi+\varphi \rho_{\mathrm{s}} / \rho_{\mathrm{f}}\right)},
$$

Eqs. (3)-(5) take the form

$$
\begin{gathered}
\dddot{F}+\frac{1}{2} F \ddot{F}=0, \\
\frac{1}{P r_{\mathrm{nf}}} \ddot{\theta}+\frac{1}{2} F \dot{\theta}=0, \\
F(0)=0, \quad \dot{F}(0)=\varepsilon, \quad \dot{F}(\infty)=1-\varepsilon,
\end{gathered}
$$

where the dots denote differentiations with respect to the new independent variable $\xi$. The temperature boundary conditions (6), on the other hand, remain unchanged. In Eq. (11), $P r_{\mathrm{nf}}$ stands for the effective Prandtl number of the nanofluid which has been defined as

$$
P r_{\mathrm{nf}}=\frac{\nu_{\mathrm{nf}}}{\alpha_{\mathrm{nf}}}=\frac{1-\varphi+\varphi \frac{\left(\rho C_{p}\right)_{\mathrm{s}}}{\left(\rho C_{p}\right)_{\mathrm{f}}}}{(1-\varphi)^{2.5}\left(1-\varphi+\varphi \frac{\rho_{\mathrm{s}}}{\rho_{\mathrm{f}}}\right)} \frac{k_{\mathrm{f}}}{k_{\mathrm{nf}}} \operatorname{Pr}_{\mathrm{f}} .
$$

The dimensionless velocity profiles $f^{\prime}(\eta)$ are obtained from the corresponding rescaled quantities $\dot{F}(\xi)$ simply as $f^{\prime}(\eta)=\dot{F}(\xi)=F(\eta / \sqrt{a})$, and the dimensionless wall shear stress $f^{\prime \prime}(0)$ and wall temperature gradient $\theta^{\prime}(0)$ of the original problems result in terms of the corresponding quantities $\ddot{F}(0)$ and $\dot{\theta}(0)$ of the rescaled problems according to

$$
f^{\prime \prime}(0)=\frac{\ddot{F}(0)}{\sqrt{a}}, \quad \theta^{\prime}(0)=\frac{\dot{\theta}(0)}{\sqrt{a}} .
$$

Now, one sees at a glance that Eqs. (10)-(12) coincide with the familiar boundary value equations of the corresponding Blasius $(\varepsilon=0)$ and Sakiadis $(\varepsilon=1)$ flow and heat transfer problems of the base fluid, with the (computationally trivial) difference that $P r$ is replaced by $P r_{\mathrm{nf}}$. Accordingly, in the limiting case of vanishing volume fraction of the nanoparticles, $\varphi \rightarrow 0$, one has $a \rightarrow 1, k_{\mathrm{f}} / k_{\mathrm{nf}} \rightarrow 1$, and thus the effective Prandtl number (13) of the nanofluid reduces to the Prandlt number $P r_{\mathrm{f}}$ of the base fluid.

The numerical solution of both the Blasius and the Sakiadis flow problems are well known and correspond to the values $\ddot{F}(0)=0.3321$ (Blasius flow) and $\ddot{F}(0)=-0.44375$ (Sakiadis flow) of the rescaled dimensionless wall shear stress, respectively. Thus, the first Eq. (7) for the skin friction coefficient goes over in

$$
\operatorname{Re}_{x}^{1 / 2} C_{f}=\frac{0.3321}{\sqrt{a}(1-\varphi)^{2.5}} \text { (Blasius), } \quad R e_{x}^{1 / 2} C_{f}=-\frac{0.44375}{\sqrt{a}(1-\varphi)^{2.5}} \text { (Sakiadis) }
$$

Consequently, in the considered nanofluid problems, neither the behavior of $f^{\prime \prime}(0)$, nor that of $\operatorname{Re}_{x}^{1 / 2} C_{f}$ requires a new special investigation. The mentioned quantities can be obtained by simple arithmetic calculations with the aid of Eqs. (14) and (15). Furthermore, Eq. (15) leads to the following relationship between the Blasius and Sakiadis skin friction coefficients:

$$
-\left(R e_{x}^{1 / 2} C_{f}\right)_{\text {Sakiadis }}=1.33619\left(\operatorname{Re}_{x}^{1 / 2} C_{f}\right)_{\text {Blasius }} .
$$

In this way, the data of Table 3 of [10] can be obtained from the corresponding data of Table 2 of [10] with the aid of the proportionality relationship (16). The same holds for Figures 1 and 3 of [10] in the sense that the curves plotted in Figure 3 result from the curves of Figure 1 by the simple formula (16). By the way, Eq. (16) also shows that several data of Table 3 of [10] are not very accurate (in general their accuracy is not higher than two decimal digits). For example, in the first line of Table 3 corresponding to $\varphi=0$ (base fluid without nanoparticles) all the values should be equal to 0.44375 , but they are not. 
Concerning the temperature boundary value problem similar simplifications occur. Indeed, the temperature solution results in terms of the known solution $F(\xi)$ of the rescaled flow problem for specified values of $P r_{\mathrm{nf}}$ and $a$ from the integral formula

$$
\theta(\eta)=1+\dot{\theta}(0) \int_{0}^{a^{-1 / 2} \eta} \exp \left(-\frac{1}{2} P r_{\mathrm{nf}} \int_{0}^{\xi_{2}} F\left(\xi_{1}\right) \mathrm{d} \xi_{1}\right) \mathrm{d} \xi_{2},
$$

where

$$
\dot{\theta}(0)=-\left[\int_{0}^{\infty} \exp \left(-\frac{1}{2} P r_{\mathrm{nf}} \int_{0}^{\xi_{2}} F\left(\xi_{1}\right) \mathrm{d} \xi_{1}\right) \mathrm{d} \xi_{2}\right]^{-1} .
$$

Therefore, one sees that compared to the case of the base fluid, the corresponding nanofluid heat transfer problem does not require any new special analysis. With the value (18) of the rescaled wall temperature gradient $\dot{\theta}(0)$, the corresponding quantity $\theta^{\prime}(0)$ of the original problems is obtained from the second of Eq. (14) and the corresponding Nusselt number from the second of Eq. (7) by simple arithmetic operations. Equation (18) of $\dot{\theta}(0)$ results in turn from the corresponding equation of the base fluid by simply replacing the value of $\operatorname{Pr}_{f}$ by the value of $P r_{\mathrm{nf}}$ given by Eq. (13). In this way,

$$
\left(R e_{x}^{-1 / 2} N u\right)_{\text {Blasius }}=-\frac{k_{\mathrm{nf}}}{k_{\mathrm{f}} \sqrt{a}}[\dot{\theta}(0)]_{\text {Blasius }}, \quad\left(R e_{x}^{-1 / 2} N u\right)_{\text {Sakiadis }}=-\frac{k_{\mathrm{nf}}}{k_{\mathrm{f}} \sqrt{a}}[\dot{\theta}(0)]_{\text {Sakiadis }} .
$$

It is also worth mentioning here that the homogeneous nanofluid models come into conflict even with the experimentally measured values of their Nusselt numbers, which exceed the predictions of the classical Dittus-Boelter correlations systematically (see Fig. 1 of [1]).

\section{Conclusions}

We may conclude therefore that the homogeneous nanofluid boundary layer heat transfer models which do not incorporate the two main velocity-slip effects of the nanoparticles with respect to the base fluid, namely the Brownian diffusion and the thermophoretic diffusion are basically equivalent to the corresponding regular models for the base fluid and can be mapped on each other by elementary scaling transformations. The only difference consists in the numerical values of the parameters present in the respective governing equations. Thus, the corresponding nanofluid results can be recovered from the solutions of already solved regular problems by simple arithmetic operations, without any additional research effort.

\section{References}

1. Buongiorno, J.: Convective transport in nanofluids. ASME J. Heat Transf. 128, 240-250 (2006)

2. Kuznetsov, A.V., Nield, D.A.: Natural convective boundary-layer flow of a nanofluid past a vertical plate. Int. J. Thermal Sci. 49, 243-247 (2010)

3. Khan, W.A., Pop, I.: Boundary-layer flow of a nanofluid past a stretching sheet. Int. J. Heat Mass Transf. 53, 2477-2483 (2010)

4. Bachok, N., Ishak, A., Pop, I.: Boundary-layer flow of nanofluids over a moving surface in a flowing fluid. Int. J. Thermal Sci. 49, 1663-1668 (2010)

5. Kuznetsov, A.V., Nield, D.A.: Double-diffusive natural convective boundary-layer flow of a nanofluid past a vertical plate. Int. J. Thermal Sci. 50, 712-717 (2011)

6. Khan, W.A., Aziz, A.: Natural convection flow of a nanofluid over a vertical plate with uniform surface heat flux. Int. J. Thermal Sci. Online First (2011)

7. Nield, D.A., Kuznetsov, A.V.: The Cheng-Minkowycz problem for natural convective boundary-layer flow in a porous medium saturated by a nanofluid. Int. J. Heat Mass Transf. 52, 5792-5795 (2009)

8. Kuznetsov, A.V., Nield, D.A.: The onset of double-diffusive nanofluid convection in a layer of a saturated porous medium. Transp. Porous Media 85, 941-951 (2010)

9. Gorla, R.S.R., Chamkha, J.A., Rashad, A.M.: Mixed convective boundary layer flow over a vertical wedge embedded in a porous medium saturated with a nanofluid: natural convection dominated regime. Nanoscale Res. Lett. 6, 207-216 (2011)

10. Ahmad, S., Rhoni, A.M., Pop, I.: Blasius and Sakiadis problems in nanofluids. Acta Mech. 218, 195-204 (2011)

11. Hamad, M.A.A.: Analytical solution of the natural convection flow of a nanofluid over a linearly stretching sheet in the presence of magnetic field. Int. Commun. Heat Mass Transf. 38, 487-492 (2011) 
12. Grosan, T., Pop, I.: Axisymmetric mixed convection boundary layer flow past a vertical cylinder in a nanofluid. Int. J. Heat Mass Transf. 54, 3139-3145 (2011)

13. Yacob, N.A., Ishak, A., Nazar, R., Pop, I.: Falkner-Skan problem for a static and moving wedge with prescribed surface heat flux in a nanofluid. Int. Commun. Heat Mass Transf. 38, 149-153 (2011)

14. Yacob N.A., Ishak A., Pop I.: Falkner-Skan problem for a static and moving wedge in a nanofluid. Int. J. Thermal Sci. 50, 133-139 (2011)

15. Yacob, N.A., Ishak, A., Pop, I., Vajravelu, K.: Boundary layer flow past a stretching/shrinking surface beneath an external uniform shear flow with a convective surface boundary condition in a nanofluid. Nanoscale Res. Lett. 6, 314-321 (2011)

16. Hamad, M.A.A., Pop, I.: Unsteady MHD free convection flow past a vertical permeable flat plate in a rotating frame of reference with constant heat source in a nanofluid. Heat Mass Transf., Online First, 15. May 2011

17. Hamad, M.A.A., Pop, I.: Scaling transformations for boundary layer flow near the stagnation-point on a heated permeable stretching surface in a porous medium saturated with a nanofluid and heat generation/absorption effects. Transp. Porous Media 37, 25-39 (2011)

18. Tiwari, R.K., Das, M.K.: Heat transfer augmentation in a two-sided lid-driven differentially heated square cavity utilizing nanofluids. Int. J. Heat Mass Transf. 50, 2002-2018 (2007) 